\title{
A GLOBAL NETWORK OF SMALL TELESCOPES AS A RESOURCE FOR ASTRONOMICAL RESEARCH AND EDUCATION
}

\author{
DAVID L. CRAWFORD \\ GNAT, Inc. \\ 2127 E. Speedway, Suite 209, Tucson Arizona 85719 USA \\ e-mail: crawford@gnat.org
}

\section{Introduction}

There is no question that relatively small telescopes are powerful tools for astronomy, just as they always have been. With the new detectors and full usage of computers, they have become even more powerful, enabling us to do with a one-meter aperture telescope today more than 4-meter or 5 -meter telescopes could do only a few decades ago. And the small ones cost a lot less to build and operate than the large ones. As such, small telescopes are the main hope for observing time for the many astronomers worldwide who need them as part of their research (or educational) tools. They can make a major impact on many areas of research and will be of great value for scientific education as well. Astronomy is very interesting to students and to the general public, not just to astronomers. Furthermore, most areas of astronomical research are data poor and more telescopes are needed to effectively attack the problems. Only a very few of us have adequate telescope time for our research or educational needs.

Technology has changed a lot over the last few decades, allowing truly a new generation of telescopes, both large and small, to be designed and put into operation. The telescopes are smaller, lower cost, and more effective than their older generation counterparts. New imaging detectors allow us to work both fainter and over a larger area than our older photometers or with traditional photographic methods. A great many individuals and organizations are beginning to take advantage of these potentials.

In addition, the advances in communication technology not only allow us to be in nearly real time effective communication with each other, but with our telescopes too. We can truly envisage a multi-user, multi-telescope, multi-site observatory. Thus small telescopes can be located at excellent, remote observing sites and operated by many users automatically.

These technology changes empower us to conceive of a global network of astronomical telescopes. In an effort to bring such a facility to reality, we have recently incorporated a non-profit organization, GNAT, Inc. The rest of this paper summarizes the goals and status of GNAT as of July 1997, and briefly discusses the potential impact on astronomy in developing countries. Creative and fertile minds and ideas exist everywhere, and need nurturing.

\section{The Technology}

There have been many improvements in telescope technology over the last few decades. We now have light weight mirror blanks, active optical surface and wavefront control, fast focal ratios for primary mirrors, smaller and lighter weight mountings, full usage of computers in telescope control and data handling, smaller and thermally more friendly housings, relatively inexpensive CCD cameras, and many other items.

It is important to note that many of the items listed above impact the design and operation of small telescopes as well as large ones, and as with the larger ones, it also means that costs are less for a given aperture than in the older generation telescopes. While the costs of telescopes scale with some relatively high exponent of the aperture (about 2.6, for example, "all things being equal"), the costs for telescopes of all apertures are much lower (in inflation adjusted currency) than previously. 
[It is interesting to compare that exponent with one that could be estimated to scale the number of users or the number of papers as a function of aperture. We leave it here to the reader to estimate the value of such exponents. It is much smaller than 2.6 , no matter what the assumptions used.] We are now seeing computer controlled, automatic and remotely operated small telescopes in routine operation at a number of observing sites. The technology is still changing rapidly, and it is sure to bode well for both large and small telescopes.

In addition, the state of art in astronomical instrumentation has improved greatly in recent years. A CCD imaging photometer is a powerful tool for a small telescope, making a one meter aperture telescope more powerful for many research problems than the 5-m telescope was only a few decades ago. It is great for spectroscopy as well, of course.

Another change in technology that has been of great benefit for astronomy has been the improvements in electronic communications, email and the Internet and the World Wide Web, for example. We now can communicate rapidly with our colleagues almost anywhere, sending data back and forth and accessing available data bases in a very efficient manner. We can also control our new generation telescopes remotely in addition to getting the data from them.

It is easy to envisage, therefore, a global network of automatic small telescopes, operated remotely from a "Homebase." Such telescopes would be very powerful tools for many important problems in astronomy, such as monitoring all sorts of variable objects, from quasars and lensing galaxies to nearby stars, and even the planets and asteroids. They would be most valuable for many sorts of surveys. They would be a critical resource for improvement in photometric systems and standard stars. Finally, they would be able to supply a good deal of observing time for all of us who find our programs to be sadly lacking in adequate observing hours.

There is also no doubt that such a system of telescopes, and the astronomers involved (all of us), would be able to make a major impact on the quality of scientific education everywhere. Images and data would be accessible to all on the Web and in hardcopy, as well as many high quality tutorials, lists of active research programs and who is involved, and so forth. It is easy to imagine that such a system, when in full operation, would be able to make a significant positive impact on well over 100 million students worldwide.

It can be done at relatively low cost; new generation small telescopes are not astronomically expensive, and they provide an essential and balanced complement to larger telescopes, to radio and space astronomy, and to education at universities and other schools. We think that GNAT can be a help in providing such facilities at a cost significantly lower than everyone "doing it themselves." It can be a big help in linking users together in many other ways as well, not duplicating nor wiping out what anyone else is doing, but being a complement and a link to all of them. A catalyst.

\section{GNAT}

It is just such considerations that have led a number of us to propose the formation of GNAT, a Global Network of Astronomical Telescopes. It has now been incorporated as a non-profit organization, with the stated goal of being a catalyst and information source for all those interested in research and education using relatively small astronomical telescopes. Over the next decade, GNAT expects to have a number of new generation small telescopes operating at several excellent observing sites worldwide. It will also be a networking contact for small telescope users with telescopes anywhere and for any individuals and organizations interested in the issues, with or without telescopes of their own.

It should be of great help in insuring homogeneous and accurate data for all. Consider, for example, just the gain that would be made by the volume purchase of high quality, matched filter sets. GNAT would not be at the cutting edge of technology development or use. It can't afford to be. It will have as a goal getting 80 percent or more of the performance of the frontier instrumentation at 20 percent or less of the cost, and with high reliability. A key item is "Value Per Cost." Astroeconomics is an important and interesting subject.

GNAT is currently operating according to its Bylaws, and it is developing a formal Business Plan. The Business Plan will also result in one or more proposals to fund GNAT new generation telescopes, based on the current state of the art in small telescope design. GNAT has been actively discussing such designs and the potentials with telescope manufacturers and with potentials users of such telescopes. The proposal will be for at least twelve telescopes located at least six observing 
sites, three in the northern hemisphere and three in the southern. Naturally, the GNAT network can and will grow in other ways, perhaps by incremental funding from different funding sources and from different countries. It should be easy to add to the initial complement of telescopes and to increase the range of telescope apertures and instrumentation. Many telescopes owned and operated by other organizations can and will also participate in GNAT activities.

We quote here from the GNAT Bylaws concerning GNAT's objectives;

1. Service to the public and to astronomy via scientific research, education, and public information concerning the development and operation of a global network of (small) astronomical telescopes for astronomical research and education, for developing national and international cooperation, and for archiving and standardizing essential astronomical data and information.

2. Service to the membership and others via collection of information, distribution of information, education on all aspects of the use of small telescopes and related topics and assistance with member's problems by sharing knowledge on a local, national, and international basis.

3. Any moneys or funds from membership, programs, or services shall be used totally for research and education, and to further the goals of GNAT. No funds shall be used for personal gain except for reimbursement of expenses for program activities.

4. GNAT has been organized exclusively for educational and scientific purposes within the meaning of Section 501(c)(3) of the United States Internal Revenue Code.

\section{Status}

While some aspects of the status of GNAT activities may be gleaned from the above discussion, it may be worth listing a few specifics here:

1. As of mid-1997, GNAT had several formal organizational members: Colorado State University, San Diego State University, the College of Charleston, University of Oregon, Grasslands Observatory, and the University of Bradford (UK). Others are pending. There are also a number of individuals who are formal members. There is a dues structure, and those interested in membership are most welcome to inquire. As of July 1997, GNAT has close to 50 official organizational and individual members in the fold.

2. An 0.5-meter telescope has been obtained under an option-to-buy arrangement with a commercial company supplying telescopes. The telescope is now in Tucson undergoing tests and software development. It has already begun a number of limited research programs, and it should be available for routine operation, including collection of data with a CCD imaging photometer, about the end of 1997. This prototype telescope, as well as others not formally part of GNAT, should supply good data and the operating experience useful both for all those interested in such new generation small telescopes and in the preparation of the Proposal for the full GNAT telescope system.

3. GNAT hosted meetings were held in Tucson in April and November 1995 to discuss the issues, and in particular the viability of standardized designs for small telescopes of several aperture sizes. We think that it is essential that such designs exist, and that they be accepted as a fact by the astronomical community, in order to obtain high quality telescopes at relatively low cost. If such is possible, we all stand to benefit greatly. The most recent meetings were held on April 20 to 22, 1996, in Ft. Collins, Colorado, with the principal topic "The Science and Education Potentials of a GNAT," and in Rarotonga (Cook Islands) and Honolulu (Hawaii) in May 1997. The announcement, agenda, and notes taken at the meetings appear on the GNAT Web site (mentioned in the next item). Additional meetings and workshops are planned. Those interested individuals who can not attend these GNAT meetings are most welcome to input their questions and feedback both before and after the meetings (via mail or the Web), or to send a paper for presentation.

4. GNAT has a World Wide Web page, which we hope will become a mechanism of information exchange for those interested in small telescopes. It is quite new, so its usefulness will grow with time, in both quality and quantity. Feedback about content, input for posting, and questions are welcome at any time. The address is: http://www.gnat.org.

5. We are in the beginning stages of preparing a Business Plan for GNAT, as well as a Proposal for twelve new generation small telescopes to be located at least six world-wide observing sites. We expect final drafts of both of these documents to be finished by June 1998. We welcome your involvement in this effort. 
6. We are now actively promoting the idea of GNAT Working Groups. These should be of great value in developing and implementing GNAT programs. The first several are now in the formative stages, including Standard Systems and Standard Stars, Open Clusters, and Photometry of Active Galaxies. We welcome input about these topics, and any others. The Working Groups will each have their own pages on the GNAT Web pages, beginning in the immediate future. We also welcome membership to these WG's, of course.

\section{Disclaimers}

In the interest of clarity, please note the following disclaimers:

1. GNAT is not a part of NOAO, KPNO, nor any other such astronomical organization. Some individuals who are involved with GNAT as part of their research activities are members of the staff of such organizations, of course, and a number of organizations are affiliated with GNAT as Members, but GNAT is a totally independent non-profit organization, as noted above.

2. Neither GNAT nor any of its Board Members have any formal or fiscal connection with any commercial company producing astronomical telescopes or instrumentation or software. We want to see any and all of them that offer a good product be successful. That will be good for astronomy and for GNAT and for all of us who hope to get quality telescopes and accessories at a good cost. As such, GNAT is and will be in contact with all of them that we know are interested in the issues and appear to offer a quality product. The GNAT Board Members involvement with GNAT is strictly as volunteers, and the Bylaws allow no compensation to the Board Members for these voluntary efforts. Naturally, GNAT and other organizations (GNAT members and others) will be from time to time signing formal contracts with commercial companies to obtain telescopes and instrumentation. All such will be by negotiation or a formal bidding process. Naturally, we also welcome any and all such commercial companies to GNAT membership, just as we do any organization.

\section{Conclusion}

There are many other aspects to the issues of small telescopes in a balanced and rational approach to astronomical research and education. We have just outlined a few of them here. We welcome your questions and your input. There is no doubt that the technology and the need and the potentials are all ripe for a viable global network of small telescopes. GNAT is one mechanism to help make it happen. A rational, coordinated approach should be of great help to the many individual efforts. There is also no doubt that it can be done, or that it should be done. It is of very low risk, of relatively low cost, and of "astronomically" high potential. The Value Per Cost ratio is enormous. We hope that all those interested individuals and organizations will join us in helping to bring GNAT to a reality. We welcome and need your active involvement, wherever you are located or whatever your research and educational interests are. Please do get involved. 